\title{
AMEGO: Active Galactic Nuclei
}

\author{
Jeremy S. Perkins* \\ NASA/GSFC \\ E-mail: jeremy.s.perkins@nasa.gov
}

Marco Ajello ${ }^{a}$, Dieter Hartmann $^{a}$, Lea Marcotulli ${ }^{a}$, Eileen Meyer ${ }^{b}$, Vaidehi S. Paliya $^{a}$, and Tonia Venters ${ }^{c}$

${ }^{a}$ Clemson University

${ }^{b}$ University of Maryland, Baltimore County

${ }^{c} N A S A / G S F C$

The AMEGO Team

https://asd.gsfc.nasa.gov/amego/

The All-Sky Medium Energy Gamma-ray Observatory (AMEGO) will survey the entire sky every three hours with its wide field of view and excellent continuum sensitivity between $200 \mathrm{keV}$ and $10 \mathrm{GeV}$. This is a prime mission to study the long-term and short-term behavior of Active Galactic Nuclei (AGN) in the MeV range. Studying AGN that have their peak power output in the $\mathrm{MeV}$ band will allow us to determine the emission mechanisms underlying these extreme phenomena. Furthermore, recent results from the Fermi Large Area Telescope have shown that MeV blazars are some of the most luminous and most distant gamma-ray AGN. A mission like AMEGO will provide the first ever census of AGN in the relatively unexplored $\mathrm{MeV}$ domain.

35th International Cosmic Ray Conference - ICRC2017

10-20 July, 2017

Bexco, Busan, Korea

${ }^{*}$ Speaker. 


\section{AMEGO}

The All-Sky Medium Energy Gamma-ray Observatory (AMEGO) is a mission in consideration as a probe for the 2020 Astrophysics Decadal review. AMEGO will survey the entire sky every three hours and see $80 \%$ of the sky every orbit with its wide field of view $(\sim 2.5 \mathrm{sr})$ and excellent continuum sensitivity between $200 \mathrm{keV}$ and $10 \mathrm{GeV}$ (see Figure 1). The AMEGO design uses well understood, well tested technologies with significant space heritage.

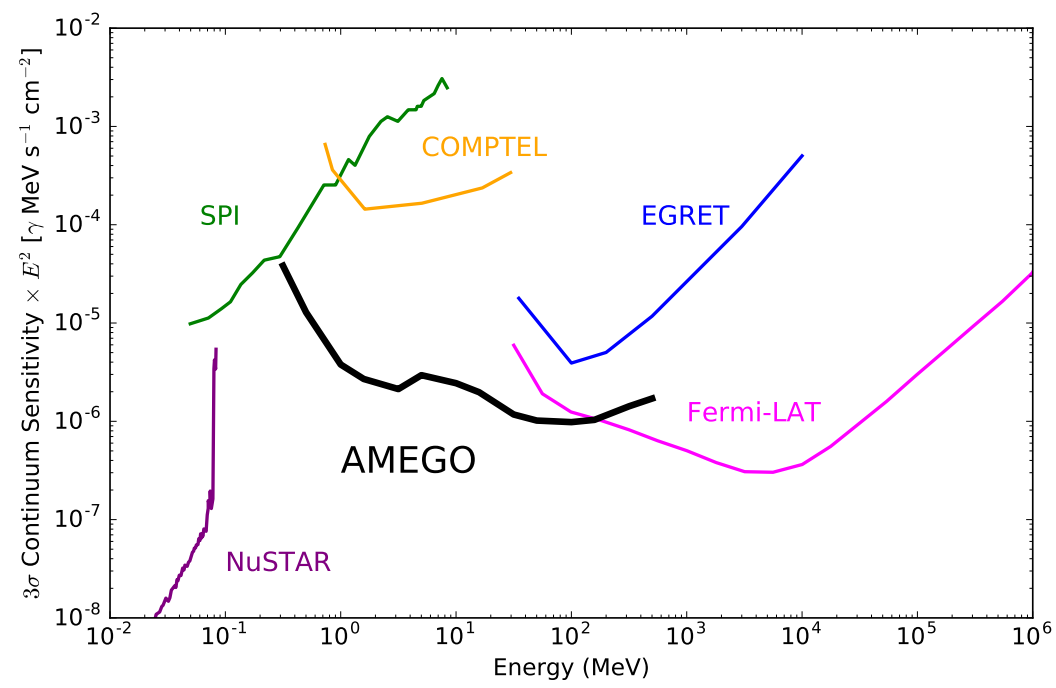

Figure 1: The simulated AMEGO $3 \sigma$ continuum sensitivity with incoming gamma-rays at an incidence angle of $37^{\circ}$. We assumed a 3-year mission or an exposure of 3 years with a $20 \%$ efficiency (due to field of view and SAA). Both the incidence angle and the efficiency were chosen to partially account for the orbit. For more details on the other curves in the figure see [1].

AMEGO will detect medium energy gamma-rays via pair production from $\sim 10 \mathrm{MeV}$ to 10 $\mathrm{GeV}$ and via Compton scattering from $200 \mathrm{keV}$ to $\sim 10 \mathrm{MeV}$. An incoming photon will undergo one of these interactions in a tracker composed of 60 layers of double sided silicon strip detectors (DSSDs) coupled to an analogue readout. This tracker will record the energies and tracks of electrons and positrons as they pass through it. A Cadmium Zinc Telluride (CZT) calorimeter is placed under the tracker as well as half way up the sides to measure the location and energy of Compton scattered photons. Finally a Cesium Iodide (CsI) calorimeter resides under the lower CZT which extends the upper energy range by measuring the energies of the particles produced via pair production and provides sensitivity to polarization and increased spectral capabilities. The full instrument is surrounded by a plastic anti coincidence detector (ACD) to veto charged particle backgrounds. For more details on the AMEGO mission see [2].

Over the past several decades the high energy (100 MeV to $100 \mathrm{GeV}$ ) sky has been surveyed by the Fermi Gamma-ray Space Telescope and several instruments on the ground such as HESS, VERITAS, MAGIC, HAWC and FACT have opened up new windows in the VHE sky (E > 100 $\mathrm{GeV})$. These missions and experiments significantly advanced gamma-ray astronomy and discovered many different types of objects. However, the medium energy gamma-ray sky has remained 
relatively unexplored (detailed measurements have been made by INTEGRAL up to $2 \mathrm{MeV}$ and the transient few $\mathrm{keV}-\sim 30 \mathrm{MeV}$ sky has been monitored by GBM while the last all-sky survey was performed by COMPTEL [3]). The MeV sky is a difficult region due to the high backgrounds and convergence of two types of detection methods (Compton and pair) but the rewards are too great to ignore and the technology proven on Fermi-LAT can be used to build a successful mission.

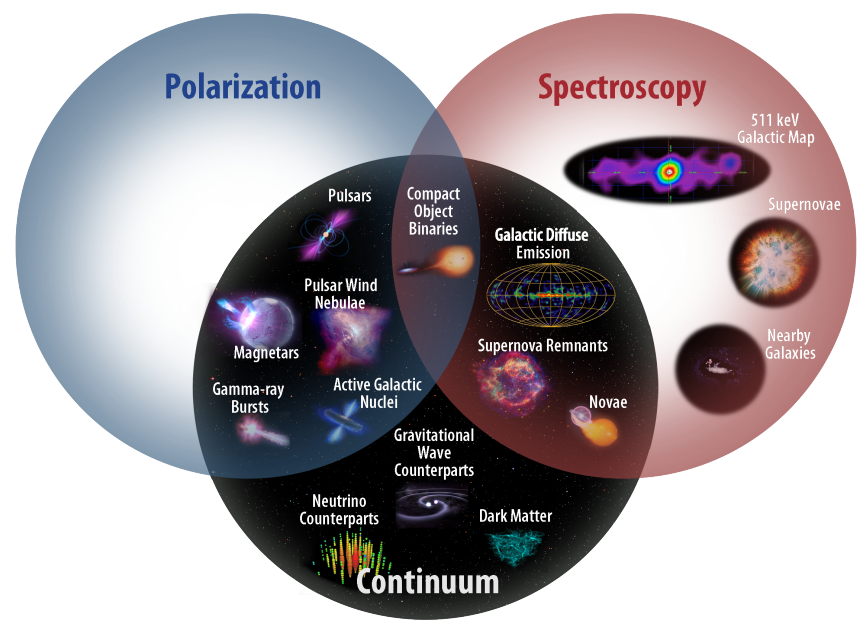

Figure 2: The AMEGO mission will observe the MeV gamma-ray sky via continuum, spectroscopy, and polarimetry. This triad of capabilities will allow us to study numerous different types of sources.

The AMEGO energy band covers transition between the thermal and non-thermal Universe. It is the only part of the electromagnetic spectrum where it is possible to directly observe nuclear processes (atomic nuclei de-excitations and excitations). It is also possible to observe gamma-ray lines due to radioactive isotopes produced in supernovae[4]. Specifically, it covers the positron annihilation line at $511 \mathrm{keV}$. Also, large populations of known sources exist with their peak power output in the $\mathrm{MeV}$ range making it crucial to study for source energetics. The AMEGO mission will initiate breakthroughs in our understanding of extreme environments.

- Astrophysical Jets: Understand the formation, evolution, and acceleration mechanisms.

- Compact Objects: Identify the physical processes in the extreme conditions.

- Dark Matter: Test models that predict signals in the MeV band.

- MeV Spectroscopy: Measure the properties of element formation in dynamic systems.

An array of objects (see Figure 2) including Pulsars and Magnetars, Gamma-ray Bursts and Multimessenger Astrophysics [5], Dark Matter [6], and Active Galaxies [these proceedings] present various versions of these phenomena. AGN are the most numerous objects in the gamma-ray sky (58\% of the Fermi-LAT catalog [7]). Many AGN seen in the LAT have their peak power output in the MeV band making them prime targets for AMEGO (§2). Additionally, the MeV band is ideal for studying radio galaxies $(\S 3)$ and Starburst Galaxies $(\$ 4)$.

Beyond understanding the jets that power these systems, AGN are cosmological probes. High redshift blazars trace massive black hole $(\mathrm{BH})$ growth at very early times and low redshift radio 
lobes can be used to measure the Extragalactic Background Light. They also play a role in structure formation and galaxy evolution. They might alter the rate of star formation [8,9], play a role in the heating of cluster gas [10] as well as heating galaxy-scale gas [11,12]. Starburst galaxies and radio loud AGN are also among the best candidates to explain the recently reported IceCube neutrino flux [13].

To fully understand AGN multi wavelengths observations are crucial and must be observed from radio to gamma-rays to be understood. They are also beamed so that it is difficult to see objects that are off-axis (the corollary to this is that we receive a huge boost for those on axis allowing us to observe $\mathrm{MeV}$ blazars to high redshifts). These beaming differences also produce synchrotron spectra peaks ranging over 6 orders of magnitude in luminosity, and more than 4 orders of magnitude in peak frequency location.

\section{MeV Blazars}

$\mathrm{MeV}$ blazars are a powerful subclass of AGN that have their inverse Compton (IC) peak below $100 \mathrm{MeV}$ (an example is shown in Figure 3). This population is not well understood and is very important to understand AGN as a population and their place in the Universe. It is clear from Fermi-LAT that there is a large population of these objects [14]. MeV blazars are among the most powerful persistent objects in the Universe. Their jet power exceeds their accretion luminosity hinting towards the importance of black hole spin. A massive BH powers these objects $\left(\geq 10^{9} M_{\odot}\right)$ and they have extremely luminous accretion disks. Interestingly they are detected up to very high redshift [15].
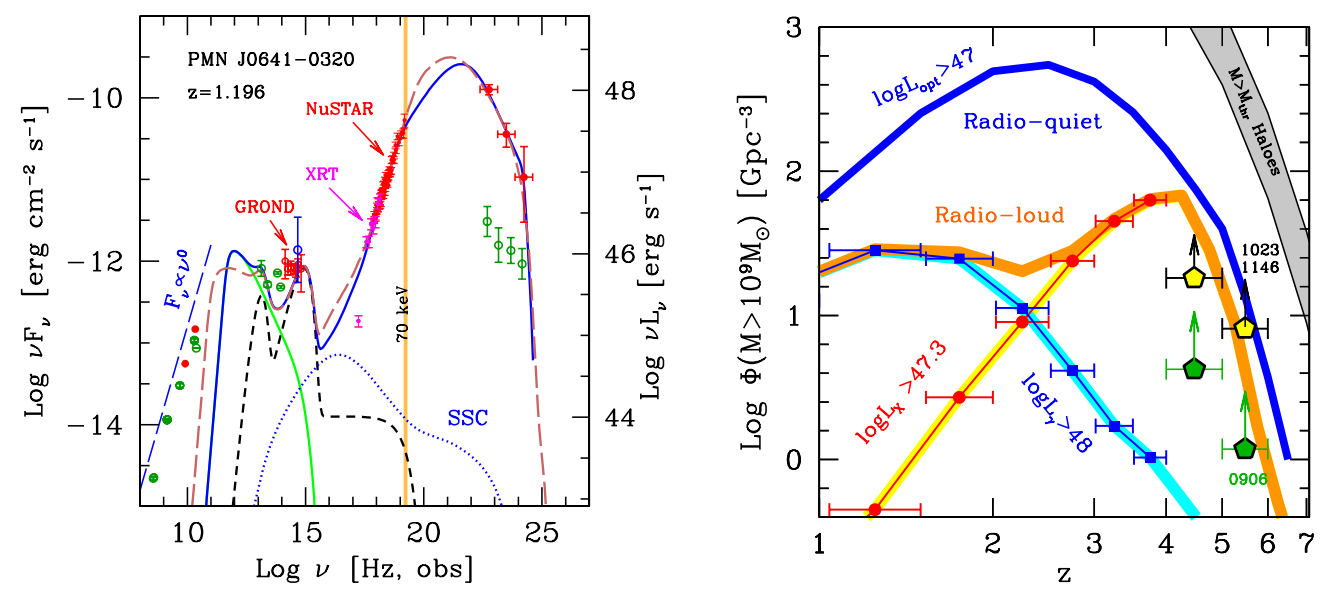

Figure 3: Left: SED of blazar PMN J0641+0320 (from [16]) with energy output peaking in the MeV energy band, shows curves that represent different models for the location of the jet energy dissipation. Right: This figure (from [17]) confirms the existence of an early peak $(\mathrm{z} \sim 4)$ of black hole formation in jetted AGN, in contrast to the main formation epoch of massive radio quiet quasars $(\mathrm{z} \sim 2.5)$.

Despite being very bright, the number of detected MeV blazars is not large. Tens have been detected by Swift/BAT [15] and a few have been discovered with NuSTAR via follow-up of LAT 
observations [18]. The limitations are the sensitivity of the BAT and the field of view of NuSTAR. Most recent efforts have been based on the improved low-energy response of the LAT (Pass 8) which resulted in the detection of five $z>3.1$ blazars. These high z objects all have large BHs $\left(M_{B H}>10^{8-9} M_{\odot}\right)$ and high Lorentz factors $(13-15)$. Every detection of a blazar implies $2 \Gamma^{2}$ objects not pointed in our direction [19] [18] has found 2 new blazars between a redshift of 3 and 4 with a $M_{B H}$ greater than $10^{9} M_{\odot}$ (only 5 of these systems where previously known) which implies the existence of 675 more objects at the same redshift. The MeV blazar population is large and an instrument like AMEGO should detect more than 100 of them up to and beyond a redshift of 5 [20]. The study of this source class will speak to SMBH growth, the disk-jet connection, radiative processes, and the $\mathrm{MeV}$ background.

$\mathrm{MeV}$ blazars have evolved more strongly than any other source class (Figure 3). Their maximum density may have occurred early in the Universe despite their large mases. It is clear that their radio loud phase may play a very important role in the growth of massive black holes. It is not understood how a black hole can grow this rapidly. It could be mergers, super Eddington accretion or a combination of both [21].

In $\mathrm{MeV}$ blazars a major fraction of the bolometric power radiates in hard $\mathrm{X}$ rays to gamma rays (Figure 3). An accurate determination of the IC peak is crucial to estimate the jet power which will yield a tighter constraint on the accretion-jet connection. The measurement of the IC peak is a missing component that will be revealed by AMEGO. Additionally the hard X-ray to MeV spectrum characterizes the seed photon field for the IC process. The source photons for the IC emission (broad-line region or IR-torus) are different depending on the energy range. The question of hadronic vs. leptonic emission could be answered in the MeV band. The models are very different in this band (emission is usually modeled as IC or proton and/or muon synchrotron) and polarization could be the determining factor.

\section{Radio Galaxies}

Similarly to MeV blazars, radio loud AGN are not well understood systems. Several fundamental open questions remain including understanding their particle content; determining their jet speeds, lifetimes, and duty cycles; understanding the connection of the Jet to the black hole; understanding the large range in morphology, jet power, and differences in particle acceleration modes between different systems; and understanding their total energetics.

For misaligned AGN their weak jets become soft in the Fermi-LAT band [22]. This is consistent with velocity gradients in the jets and puts many of these AGN below the detection threshold of the LAT and means that there are very few misaligned jets with well-measured Compton peaks.

Similarly only two systems with giant radio lobes have been measured in the gamma-ray; Cen A [23] and Fornax A [24]. These objects span large distances and are produced by plasma slowing down outside of the jet built up over the lifetime of the source. There is clear evidence that jets disturb and transfer energy to the inter cluster medium (ICM) [25]. Lower limits on the energy can be estimated from thermodynamic work needed to inflate cavities seen in X-rays [26]. Open questions remain about their particle content, the total energetics (equipartition might not be valid) and exactly how energy is transferred to the ICM. Resolving Fornax A with AMEGO would allow a measurement of the CMB [24]. 


\section{Starburst Galaxies}

At $\mathrm{MeV}$ energies the emission from starburst galaxies is diffused throughout the galaxy and not concentrated at the center or produced via jet activity (although some starburst galaxies might also harbor an AGN). This emission arises from cosmic ray interactions with gas and starlight. By studying starbursts in the $\mathrm{MeV}$ band we can learn about cosmic ray propagation and interactions.

A starburst galaxy provides a unique laboratory to study the production and propagation of cosmic rays. We have been doing this for over a century in our own galaxy but have been hampered by being embedded within it. By looking outside of our galaxy we can use the broadband spectrum from $\mathrm{X}$-rays to $\mathrm{TeV}$ gamma-rays to determine the underlying distribution of the cosmic rays. We have been looking at these object throughout the electromagnetic spectrum but the $\mathrm{MeV}$ band has been missing and is critical for constraining models (Figure 4). Open questions remain regarding cosmic ray acceleration and propagation throughout the galaxy and outside of it, and the emission processes that result in the broadband non-thermal diffuse spectrum from X-rays to gamma rays. Also at issue is whether cosmic rays play a role in star formation and their impact on the galactic environment. Specifically, the study of starburst galaxies at $\mathrm{MeV}$ energies can shed light on cosmic ray interactions and diffusion altering the nature of gas, gas heating and the regulation of star formation, impacts on chemical abundances in the ISM, diffusion altering magnetic fields, driving magnetic winds, effects on equipartition, and escape (via diffusion or advection via magnetic winds). Each of these questions is interesting in its own right but overall the study of cosmic rays in starburst galaxies via $\mathrm{MeV}$ observations is interesting because these objects are so different from our own local environment and our own galaxy. They have higher gas densities, stronger magnetic fields, and much higher star formation and supernova rates. This is a unique opportunity to study the role cosmic rays play in shaping their environment.
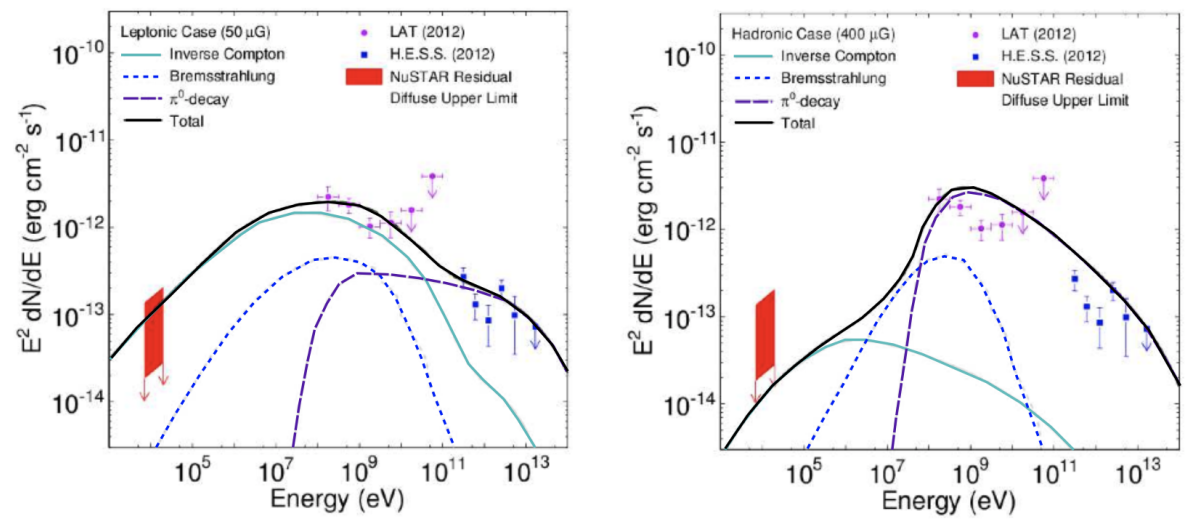

Figure 4: Broadband spectra of NGC253 showing the lack of information in the MeV band. MeV observations will disentangle if the emission is Leptonic (left) or Hadronic (right). Figure from [27].

Aside from the diffuse emission in starburst galaxies, radioactive isotopes such as ${ }^{26} \mathrm{Al}$ and ${ }^{60} \mathrm{Fe}$ are produced and injected into the ISM by massive stars. These isotopes have lifetimes comparable to the lifetimes of $\mathrm{OB}$ associations and we expect to see line emission from these isotopes in regions where massive stars have formed recently. It is also interesting to look for the ${ }^{44} \mathrm{Ti}$ pro- 
duced in SN explosions, $511 \mathrm{keV}$ from positron annihilation and other Type Ia supernova lines such at ${ }^{56} \mathrm{Ni}$ and its daughter isotope ${ }^{56} \mathrm{Co}$ [4].

\section{Summary}

The study of AGN at MeV energies will enhance our understanding of these powerful objects. A new instrument that is sensitive in the $\mathrm{MeV}$ energy range with an order of magnitude better angular resolution than previous instruments is needed. AMEGO, which has a projected energy and angular resolution that will increase sensitivity by a factor of 20-50 over previous instruments and a new capability of measuring polarisation in the gamma-ray band, will allow us to explore $\mathrm{MeV}$ blazars, starburst galaxies, radio galaxies and other AGN with a completely new perspective.

\section{References}

[1] R. Caputo, F. Kislat and J. Racusin, AMEGO: Simulations of the Instrument Performance, in 35th International Cosmic Ray Conference (ICRC2017), vol. 35 of International Cosmic Ray Conference, p. 8, July, 2017.

[2] A. Moiseev, All-Sky Medium Energy Gamma-ray Observatory (AMEGO), in 35th International Cosmic Ray Conference (ICRC2017), vol. 35 of International Cosmic Ray Conference, p. 8, July, 2017.

[3] V. Schönfelder, H. Aarts, K. Bennett, H. de Boer, J. Clear, W. Collmar et al., Instrument description and performance of the Imaging Gamma-Ray Telescope COMPTEL aboard the Compton Gamma-Ray Observatory, ApJS 86 (June, 1993) 657-692.

[4] R. Diehl, D. H. Hartmann and N. Prantzos, eds., Astronomy with Radioactivities, vol. 812 of Lecture Notes in Physics, Berlin Springer Verlag, 2011. 10.1007/978-3-642-12698-7.

[5] J. Racusin and A. Lien, AMEGO: Transients and Multi-Messenger Sources, in 35th International Cosmic Ray Conference (ICRC2017), vol. 35 of International Cosmic Ray Conference, p. 8, July, 2017.

[6] R. Caputo, M. Meyer and M. A. Sánchez-Conde, AMEGO: Dark Matter, in 35th International Cosmic Ray Conference (ICRC2017), vol. 35 of International Cosmic Ray Conference, p. 8, July, 2017.

[7] F. Acero, M. Ackermann, M. Ajello, A. Albert, W. B. Atwood, M. Axelsson et al., Fermi Large Area Telescope Third Source Catalog, ApJS 218 (June, 2015) 23, [1501.02003].

[8] N. P. H. Nesvadba, M. D. Lehnert, C. De Breuck, A. M. Gilbert and W. van Breugel, Evidence for powerful AGN winds at high redshift: dynamics of galactic outflows in radio galaxies during the "Quasar Era", A\&A 491 (Nov., 2008) 407-424, [0809.5171].

[9] M. J. Page, M. Symeonidis, J. D. Vieira, B. Altieri, A. Amblard, V. Arumugam et al., The suppression of star formation by powerful active galactic nuclei, Nature 485 (May, 2012) 213-216, [1310.4147].

[10] B. R. McNamara and P. E. J. Nulsen, Heating Hot Atmospheres with Active Galactic Nuclei, ARA\&A 45 (Sept., 2007) 117-175, [0 709.2152$].$

[11] M. D. Lehnert, C. Tasse, N. P. H. Nesvadba, P. N. Best and W. van Driel, The Na D profiles of nearby low-power radio sources: jets powering outflows, A\&A 532 (Aug., 2011) L3, [1107.1037]. 
[12] P. Guillard, F. Boulanger, G. Pineau des Forêts, E. Falgarone, A. Gusdorf, M. E. Cluver et al., Turbulent Molecular Gas and Star Formation in the Shocked Intergalactic Medium of Stephan's Quintet, ApJ 749 (Apr., 2012) 158, [1202.2862].

[13] M. G. Aartsen, K. Abraham, M. Ackermann, J. Adams, J. A. Aguilar, M. Ahlers et al., A Combined Maximum-likelihood Analysis of the High-energy Astrophysical Neutrino Flux Measured with IceCube, ApJ 809 (Aug., 2015) 98, [1507.03991].

[14] M. Ackermann, M. Ajello, A. Allafort, E. Antolini, W. B. Atwood, M. Axelsson et al., The Second Catalog of Active Galactic Nuclei Detected by the Fermi Large Area Telescope, ApJ 743 (Dec., 2011) 171, [1108.1420].

[15] M. Ajello, L. Costamante, R. M. Sambruna, N. Gehrels, J. Chiang, A. Rau et al., The Evolution of Swift/BAT Blazars and the Origin of the MeV Background, ApJ 699 (July, 2009) 603-625, [0905.0472].

[16] M. Ajello, G. Ghisellini, V. S. Paliya, D. Kocevski, G. Tagliaferri, G. Madejski et al., NuSTAR, Swift, and GROND Observations of the Flaring MeV Blazar PMN J0641-0320, ApJ 826 (July, 2016) 76, [1602.06446].

[17] T. Sbarrato, G. Ghisellini, G. Tagliaferri, L. Foschini, M. Nardini, F. Tavecchio et al., Blazar candidates beyond redshift 4 observed by Swift, MNRAS 446 (Jan., 2015) 2483-2489, [1410.0364].

[18] T. Sbarrato, G. Tagliaferri, G. Ghisellini, M. Perri, S. Puccetti, M. Baloković et al., NuSTAR Detection of the Blazar B2 1023+25 at Redshift 5.3, ApJ 777 (Nov., 2013) 147, [1309.3280].

[19] L. Marcotulli, V. S. Paliya, M. Ajello, A. Kaur, D. H. Hartmann, D. Gasparrini et al., High-redshift Blazars through NuSTAR Eyes, ApJ 839 (Apr., 2017) 96, [1703.10657].

[20] Y. Inoue, Y. T. Tanaka, H. Odaka, A. Takada, Y. Ichinohe, S. Saito et al., Prospect for future MeV gamma-ray active galactic nuclei population studies, PASJ 67 (Aug., 2015) 76, [1503.03152].

[21] S. Bonoli and L. Mayer, Forming Massive Black Hole Seeds in Galaxy Mergers, in SnowPAC 2013 Black Hole Fingerprints: Dynamics, Disruptions and Demographics, p. 3, Mar., 2013.

[22] E. T. Meyer, G. Fossati, M. Georganopoulos and M. L. Lister, Breaking the Blazar Sequence: A New View of Radio Loud AGN Unification, ArXiv e-prints (May, 2012) , [1205. 0794].

[23] A. A. Abdo, M. Ackermann, M. Ajello, W. B. Atwood, L. Baldini, J. Ballet et al., Fermi Gamma-Ray Imaging of a Radio Galaxy, Science 328 (May, 2010) 725, [1 006.3986 ].

[24] M. Ackermann, M. Ajello, L. Baldini, J. Ballet, G. Barbiellini, D. Bastieri et al., Fermi Large Area Telescope Detection of Extended Gamma-Ray Emission from the Radio Galaxy Fornax A, ApJ 826 (July, 2016) 1, [1606.04905].

[25] L. Bîrzan, D. A. Rafferty, B. R. McNamara, M. W. Wise and P. E. J. Nulsen, A Systematic Study of Radio-induced X-Ray Cavities in Clusters, Groups, and Galaxies, ApJ 607 (June, 2004) 800-809, [astro-ph/0402348].

[26] M. J. Hardcastle and M. G. H. Krause, Numerical modelling of the lobes of radio galaxies in cluster environments, MNRAS 430 (Mar., 2013) 174-196, [1301.2531].

[27] D. R. Wik, B. D. Lehmer, A. E. Hornschemeier, M. Yukita, A. Ptak, A. Zezas et al., Spatially Resolving a Starburst Galaxy at Hard X-Ray Energies: NuSTAR, Chandra, and VLBA Observations of NGC 253, ApJ 797 (Dec., 2014) 79, [1411.1089]. 